\title{
A Compositional Model for Confluent Dynamic Data-Flow Networks
}

\author{
Frank S. de Boer ${ }^{1}$ and Marcello M. Bonsangue ${ }^{2}$ \\ ${ }^{1}$ Utrecht University, The Netherlands \\ frankb@cs.uu.nl \\ ${ }^{2}$ CWI, Amsterdam, The Netherlands \\ Marcello.Bonsangue@cwi.nl
}

\begin{abstract}
We introduce a state-based language for programming dynamically changing networks which consist of processes that communicate asynchronously. For this language we introduce an operational semantics and a notion of observable which includes both partial correctness and absence of deadlock. Our main result is a compositional characterization of this notion of observable for a confluent sub-language.
\end{abstract}

\section{Introduction}

The goal of this paper is to develop a compositional semantics of a confluent subset of the language $\mathrm{MaC}$ (Mobile asynchronous Channels). $\mathrm{MaC}$ is an imperative programming language for describing the behavior of dynamic networks of asynchronously communicating processes.

A program in $M a C$ consists of a (finite) number of generic process descriptions. Processes can be created dynamically and have an independent activity that proceeds in parallel with all the other processes in the system. They possess some internal data, which they store in variables. The value of a variable is either an element of a predefined data type or it is a reference to a channel. The variables of one process are not accessible to other processes. The processes can interact only by sending and receiving messages asynchronously via channels which are (unbounded) FIFO buffers. A message contains exactly one value; this can be a value of some given data type, like integer or a boolean, or it can be a reference to a channel. Channels are created dynamically. In fact, the creation of a process consists of the creation of a channel which connects it with its creator. This channel has a unique identity which is initially known only to the created process and its creator. As with any channel, the identity of this initial channel too can be communicated to other processes via other channels. Thus we see that a system described by a program in the language $M a C$ consists of a dynamically evolving network of processes, which are all executing in parallel, and which communicate asynchronously via mobile channels. In particular, this means that the communication structure of the processes, i.e. which processes are connected by which channels, is completely dynamic, without any regular structure imposed on it a priori. 
For $\mathrm{MaC}$ we first introduce a simple operational semantics and the following notion of observable. Let $\Sigma$ denote the set of (global) states. A global state specifies, for each existing process, the values of its variables, and, for each existing channel, the contents of its buffer. The semantics $\mathcal{O}$ assigns to each program $\rho$ in $M a C$ a partial function in $\Sigma \rightarrow \mathcal{P}(\Sigma)$ such that $\mathcal{O}(\rho)(\sigma)$ collects all final results of successfully terminating computations in $\sigma$, if $\rho$ does not have a deadlocking computation starting from $\sigma$. Otherwise, $\mathcal{O}(\rho)(\sigma)$ is undefined.

This notion of observable $\mathcal{O}$ provides a semantic basis for the following interpretation of a correctness formula $\{\phi\} \rho\{\psi\}$ in Hoare logic: every execution of program $\rho$ in a state which satisfies the assertion $\phi$ does not deadlock and upon termination the assertion $\psi$ will hold. An axiomatization of this interpretation of correctness formulas thus requires a method for proving absence of deadlock.

In this paper we identify a confluent sub-language of $M a C$ which allows to abstract from the order between the communications of different processes and the order between the communications on different channels within a process $[14$, 15]. A necessary condition for obtaining a confluent sub-language is the restriction to local non-determinism and to channels which are uni-directional and one-to-one. In a dynamic network of processes the restriction to such channels implies that at any moment during the execution of a program for each existing channel there are at most two processes whose internal data contain a reference to it; one of these processes only may use this reference for sending values and the other may use this reference only for receiving values.

For confluent $M a C$ programs we develop a compositional characterization of the semantics $\mathcal{O}$. It is based on the local semantics of each single process, which includes information about the channels it has created and, for each known channel, information about the sequence of values the process has sent or read. Information about the deadlock behavior of a process is given in terms of a singleton ready set including a channel reference. As such we do not have any information about the order between the communications of a process on different channels and the order between the communications of different processes. In general, this abstraction will in practice simplify reasoning about the correctness of distributed systems.

Comparison with related work: The language $M a C$ is a sub-language of the one introduced in [3]. The latter is an abstract core for the Manifold coordination language [4]. The main feature relevant in this context is anonymous communication, in contrast with parallel object-oriented languages and actor languages, as studied, for example, in [5] and [1], where communication between the processes, i.e., objects or actors, is established via their identities.

In contrast to the $\pi$-calculus [16] which constitutes a process algebra for mobility, our language $M a C$ provides a state-based model for mobility. As such our language provides a framework for the study of the semantic basis of assertional proof methods for mobility. $M a C$ can also be seen as a dynamic version of asynchronous CSP [14]. In fact, the language $M a C$ is similar to the verification modeling language Promela [12], a tool for analyzing the logical consistency of distributed systems, specifically of data communication protocols. However, the 
semantic investigations of Promela are performed within the context of temporal logic, whereas $M a C$ provides a semantic basis for Hoare logics.

Our main result can also be viewed as a generalization of the compositional semantics of Kahn (data-flow) networks [13] (where the number of processes and the communication structure is fixed). Instead of a function the communication behavior of a process in the language $M a C$ is specified in terms of a relation between the sequence of values it inputs and the sequence of values it outputs. This information suffices because of the restriction to confluent programs. Confluence has been studied also in the context of concurrent constraint programming [9] where mobility is modeled in terms of logical variables.

Generalization of Kahn (data-flow) networks for describing dynamically reconfigurable or mobile networks have also been studied in [6] and [11] using the model of stream functions. In this paper we study a different notion of observable which includes partial correctness and absence of deadlock. Furthermore, our language includes both dynamic process and channel creation. On the other hand, we restrict to confluent dynamic networks.

\section{Syntax and Operational Semantics}

A program in the language $M a C$ is a (finite) collection of generic process descriptions. Such a generic process description consists of an association of a unique name $P$, the so-called process type, with a statement describing generically the behavior of its instances.

The statement associated with a process type $P$ is executed by a process, i.e. an instance of that process type. Such a process acts upon some internal data that are stored in variables. The variables of a process are private, i.e., the data stored in the variables of a process is not accessible by another process, even if both processes are of the same type. We denote by $V a r$, with its typical elements $x, y, \ldots$, the set of variables. The value of a variable can be either an element of a predefined data type, like integer or boolean, or a reference to a channel.

We have the following repertoire of basic actions of a process:

$$
x:=e \quad x:=\operatorname{new}(P) \quad x ! y \quad x ? y
$$

The execution of an assignment $x:=e$ by a process consists of assigning the value resulting from evaluation of the expression $e$ to the variable $x$ (we abstract here from the internal structure of $e$ and assume that its evaluation is deterministic and always terminates).

The execution of the statement $x:=\operatorname{new}(P)$ by a process consists of the creation of a new process of type $P$ and a new channel which, initially, forms a link between the two (creator and created) processes. A reference to this channel will be stored in the variable $x$ of the creator and to a distinguished variable $c h n$ of the created process. The newly created process starts executing the statement associated with $P$ in parallel with all the other existing processes.

Processes can interact only by sending and receiving messages via channels. A message contains exactly one value; this can be of any type, including channel 
references. We restrict in this paper to asynchronous channels that are implemented by (unbounded) FIFO buffers. The execution of the output action $x ! y$ sends the value stored in the variable $y$ to the channel referred to by the variable $x$. The execution of the input action $x ? y$ suspends until a value is available through the specified channel. The value read is removed from the channel and then stored in the variable $y$.

The set of statements, with typical element $S$, is generated by composing the above basic actions using well-known sequential non-deterministic programming constructs [8]. A program $\rho$ is a finite collection of generic process descriptions of the form $P \Leftarrow S$. The execution of a program $\left\{P_{0} \Leftarrow S_{0}, \ldots, P_{n} \Leftarrow S_{n}\right\}$ starts with the execution of a root-process of type $P_{0}$.

Next we define formally the (operational) semantics of the programming language by means of a transition system. We assume given an infinite set $C$ of channel identities, with typical elements $c, c^{\prime}, \ldots$. The set $V a l$, with typical elements $u, v, \ldots$, includes the set $C$ of channel identities and the value $\perp$ which indicates that a variable is 'uninitialized'.

A global state $\sigma$ of a network of processes specifies the existing channels, that is, the channels that have already been created, and the contents of their buffers. Formally, $\sigma$ is a partial function in $C \rightarrow V^{*} l^{*}$ (here $\mathrm{Val}^{*}$ denotes the set of finite sequences of elements in $\mathrm{Val}$ ). Its domain $\operatorname{dom}(\sigma) \subseteq C$ is a finite set of channel identities, representing those channels which have been created. Moreover, for every existing channel $c \in \operatorname{dom}(\sigma)$, the contents of its buffer is specified by $\sigma(c) \in V a l^{*}$. On the other hand, the internal state $s \in V a r \rightarrow V a l$ of a process simply specifies the values of its variables.

The behavior of a network of processes is described in terms of a transition relation between configurations of the form $\langle X, \sigma\rangle$, where $\sigma$ is the global state of the existing channels and $X$ is a finite multiset of pairs of the form $(S, s)$, for some internal state $s$ and statement $S$. A pair of the form $(S, s)$ denotes an active process within the network: its current internal state is given by $s$ and $S$ denotes the statement to be executed. We have the following transitions for the basic actions (we assume given a program $\rho$ ). Below the operation of multiset union is denoted by $\uplus$ and by nil we denote the empty statement.

Assignment: $\langle X \uplus\{(x:=e, s)\}, \sigma\rangle \rightarrow\langle X \uplus\{($ nil, $s[s(e) / x])\}, \sigma\rangle$, where $s(e)$ denotes the value of $e$ in $s$ and $s[v / x]$ denotes the function mapping $x$ to $v$ and otherwise acting as $s$.

Creation: $\langle X \uplus\{(x:=\operatorname{new}(P), s)\}, \sigma\rangle \rightarrow\left\langle X \uplus\left\{(\right.\right.$ nil, $\left.\left.s[c / x]),\left(S, s_{0}[c / c h n]\right)\right\}, \sigma^{\prime}\right\rangle$, where $P \Leftarrow S$ occurs in $\rho$ and $\sigma^{\prime}=\sigma[\varepsilon / c]$ for some $c \in C \backslash \operatorname{dom}(\sigma)$. Thus $\sigma^{\prime}$ extends $\sigma$ by mapping the new channel $c$ to the empty sequence $\varepsilon$. Moreover, the initial state of the newly created process $s_{0}$ satisfies the following: $s_{0}(x)=\perp$, for every variable $x \in \operatorname{Var}$. Note that the new channel $c$ forms a link between the two processes. The statement $S$ is the one associated with the process type $P$ in the program $\rho$.

Input: Let $s(x)=c(\neq \perp) .\langle X \uplus\{(x ? y, s)\}, \sigma\rangle \rightarrow\left\langle X \uplus\{(\right.$ nil, $\left.s[u / y])\}, \sigma^{\prime}\right\rangle$, where $\sigma(c)=w \cdot u$ for some $u \in V a l$, and $\sigma^{\prime}$ results from $\sigma$ by removing $u$ from the buffer of $c$, that is, $\sigma^{\prime}=\sigma[w / c]$. 
Output: Let $s(x)=c(\neq \perp)$ in $\langle X \uplus\{(x ! y, s)\}, \sigma\rangle \rightarrow\left\langle X \uplus\{(\right.$ nil, $\left.s)\}, \sigma^{\prime}\right\rangle$, where $\sigma^{\prime}$ results from $\sigma$ by adding the value $s(y)$ to the sequence $\sigma(c)$, that is, $\sigma^{\prime}=$ $\sigma[s(y) \cdot \sigma(c) / c]$.

The remaining transition rules for compound statements are standard and therefore omitted. By $\rightarrow^{*}$ we denote the reflexive transitive closure of $\rightarrow$ and $\langle X, \sigma\rangle \Rightarrow \delta$ indicates the existence of a deadlocking computation starting from $\langle X, \sigma\rangle$, that is, $\langle X, \sigma\rangle \rightarrow^{*}\left\langle X^{\prime}, \sigma^{\prime}\right\rangle$ with $X^{\prime}$ containing at least one pair $(S, s)$ such that $S \neq$ nil, and from the configuration $\left\langle X^{\prime}, \sigma^{\prime}\right\rangle$ no further transition is possible. Moreover, $\langle X, \sigma\rangle \Rightarrow\left\langle X^{\prime}, \sigma^{\prime}\right\rangle$ indicates a successfully terminating computation with final configuration $\left\langle X^{\prime}, \sigma^{\prime}\right\rangle$, that is, $\langle X, \sigma\rangle \rightarrow^{*}\left\langle X^{\prime}, \sigma^{\prime}\right\rangle$ and $X^{\prime}$ contains only pairs of the form (nil, $s$ ).

We are now in a position to introduce the following notion of observable.

Definition 1. Let $\rho=\left\{P_{0} \Leftarrow S_{0}, \ldots, P_{n} \Leftarrow S_{n}\right\}$ be a program. By $\left\langle X_{0}, \sigma_{0}\right\rangle$ we denote its initial configuration $\left\langle\left\{\left(S_{0}, s_{0}\right)\right\}, \sigma_{0}\right\rangle$, where $s_{0}(x)=\perp$, for every variable $x$, and $\operatorname{dom}\left(\sigma_{0}\right)=\emptyset$. We define

$$
\mathcal{O}(\rho)= \begin{cases}\delta & \text { if }\left\langle X_{0}, \sigma_{0}\right\rangle \Rightarrow \delta \\ \left\{\langle X, \sigma\rangle \mid\left\langle X_{0}, \sigma_{0}\right\rangle \Rightarrow\langle X, \sigma\rangle\right\} & \text { otherwise }\end{cases}
$$

Note that thus $\mathcal{O}(\rho)=\delta$ indicates that $\rho$ has a deadlocking computation. On the other hand, if $\rho$ does not have a deadlocking computation then $\mathcal{O}(\rho)$ collects all the final configurations of successfully terminating computations.

\section{Compositionality}

[n this section we introduce, for a certain kind of programs, a compositional sharacterization of the notion of observable defined in the previous section.

First of all we restrict to local non-determinism. Moreover, we assume now a typing of the variables: we have variables of some predefined data types and we assume channel variables to be either of type $\iota$, for input, and $o$, for output. Let $\bar{C}$, with typical element $\bar{C}$, be a copy of $C$. A channel variable of type $\iota$ always refers to an element of $C$, whereas, a channel variable of type $O$ always refers to an element of $\bar{C}$. (The set of all possible values thus includes both $C$ and $\bar{C}$.) We restrict to programs which are well-typed. In particular, in an output $x ! y$ the variable $x$ is of type $o$ and in an input $x ? y$ the variable $x$ is of type ८. An input $x ? y$ now also suspends if the value to be read is not of the same type as the variable $y$. Moreover, we assume that the distinguished variable $c h n$ (used for storing the initial link with the creator) is of type $o$. Consequently, in $x:=\operatorname{new}(P)$ the variable $x$ has to be of type $\iota$. In other words, initially, the flow of information along the newly created channel goes from the created to the creator process.

Finally, we assume that an output $x ! y$, where $y$ is a channel variable, is immediately followed by an assignment which uninitializes the variable $y$, i.e. it sets $y$ to $\perp$. But for this latter, we do not allow channel variables (either of type $\iota$ or 0 ) to appear in an assignment. As a result, channels are one-to-one and uni-directional. 
We extend now the notion of an internal state $s$ to include the following information about the channels. Let $\gamma \notin V a l$ and $V a l_{\gamma}=V a l \cup\{\gamma\}$. For each channel $c \in C, s(c) \in V a l_{\gamma}^{*}$ denotes, among others, the sequence of values received from channel $c$, and $s(\bar{c}) \in V a l_{\gamma}^{*}$, denotes, among others, the sequence of values sent along channel $c$. More precisely, in a sequence $w_{1} \cdot \gamma \cdot w_{2} \cdot \gamma \cdot \cdots$, the symbol $\gamma$ indicates that first the sequence of values $w_{1}$ has been sent along $c$ (or received from $c$ ) and that after control over this channel has been released and subsequently regained again the sequence $w_{2}$ has been sent (or received), etc.. Note that a process releases control over a channel only when it outputs that channel and that it subsequently may again regain control over it only by receiving it via some input.

Additionally, we introduce a component $s(\nu) \in(C \cup\{\perp\}) \times \mathcal{P}(C)$. The first element of $s(\nu)$ indicates the channel which initially links the process with its creator (in case of the root-process we have here $\perp$ ). The second element of $s(\nu)$ indicates the set of channels which have been created by the process itself.

Given this extended notion of an internal state of a process we now present the transitions describing the execution of the basic actions with respect to the internal state of a process (we omit the standard transition for a simple assignment).

Creation: Let $s(\nu)=(u, V)$ and $c \notin V$ in $\langle x:=\operatorname{new}(P), s\rangle \rightarrow\left\langle\right.$ nil, $\left.s^{\prime}[c / x]\right\rangle$. Here $s^{\prime}$ results from $s$ by adding $c$, that is, $s^{\prime}(\nu)=(u, V \cup\{c\})$. The only effect at the local level of the execution of a basic action $x:=\operatorname{new}(P)$ is the assignment to $x$ of a channel $c$ which is new with respect to the set of channels already created by the process.

Output 1: If $s(x)=\bar{c}$ and $y$ is not a channel variable, i.e. $y$ is of some given data type like the integers or booleans, then $\langle x ! y, s\rangle \rightarrow\langle$ nil, $s[s(\bar{c}) \cdot s(y) / \bar{c}]\rangle$. The local effect of an output (of a value of some predefined data type) consists of adding the value stored in the variable $y$ to the sequence of values already sent.

Output 2: If $s(x)=\bar{c}$ then $\langle x ! y, s\rangle \rightarrow\langle$ nil, $s[s(\bar{c}) \cdot v / \bar{c}][s(v) \cdot \gamma / v]\rangle$, where $v=s(y)$ and $y$ is a channel variable. So after the output along the channel $c$ of the value $v$ stored in the variable $y$, first the value $v$ is appended to $s(\bar{c})$, which basically records the sequence of values sent along the channel $\bar{c}$. Finally, the output of channel $v$ (and consequently its release) is recorded as such by $\gamma$ in the sequence $s(v)$ which records the sequence of values sent along the channel $v$, in case $v \in \bar{C}$, and received from it, in case $v \in C$. Note that we have to perform the state-changes indicated by $[s(\bar{c}) \cdot v / \bar{c}]$ and $[s(v) \cdot \gamma / v]$ in this order to describe correctly the case that $v=\bar{c}$.

Input: If $s(x)=c(\neq \perp)$ then $\langle x ? y, s\rangle \rightarrow\langle$ nil, $s[v / y, s(c) \cdot v / c]\rangle$, where $v \in V a l$ is an arbitrary value (of the same type as $y$ ). This value is assigned to $y$ and appended to the sequence $s(c)$ of values received so far (along channel $c$ ). Note that because channels are one-to-one and unidirectional it cannot be the case that $v=c$.

On the basis of the above transition system (we omit the rules from compound statement since they are standard) we define the operational semantics of statements as follows. 
Definition 2. An (extended) initial state $s$ satisfies the following: for some $u \in$ $C \cup\{\perp\}$ we have that $s(\operatorname{chn})=u$, and $s(x)=\perp$, for every other variable, moreover, $s(d)=s(\bar{d})=\epsilon$, for every channel $d$, and, finally, $s(\nu)=(u, \emptyset)$. We define $\mathcal{O}(S)=\langle T, R\rangle$, where $T=\left\{s^{\prime} \mid\langle S, s\rangle \rightarrow^{*}\left\langle\right.\right.$ nil, $\left.s^{\prime}\right\rangle$ for some initial state $s\}$ and $R=\left\{\left(s^{\prime}, s(x), t(y)\right) \mid\langle S, s\rangle \rightarrow^{*}\left\langle x ? y ; S^{\prime}, s^{\prime}\right\rangle\right.$, for some initial state $\left.s\right\}$ (here $t(y)$ denotes the type of $y$ ).

The component $T$ in the semantics $\mathcal{O}(S)$ collects all the final states of successfully terminating (local) computations of $S$ (starting from an initial state). The component $R$, on the other hand, collects all the intermediate states where control is about to perform an input, plus information about the channel involved and the type of the value to be read. The restriction to local non-determinism implies that when an input $x ? y$ is about to be executed, it will always appear in a context of the form $x ? y ; S$ for some (possibly empty) statement $S$ (no other inputs are offered as an alternative).

The information in $R$ corresponds with the well-known concept of the ready sets [17] and will be used for determining whether a program (containing a process type $P \Leftarrow S$ ) has a deadlocking computation.

Our compositional semantics is based on the compatibility of a set of internal states (without loss of generality we may indeed restrict to sets rather than multisets of extended internal states $s$ because of the additional information $s(\nu)$ ). In order to define this notion we use the set $C_{\perp}=C \cup\{\perp\}$, ranged over by $\alpha, \beta, \ldots$, to identify processes. The idea is that the channel which initially links the created process with its creator will be used to identify the created process itself ( $\perp$ will be used to identify the root-process). We use these process identifiers in finite sequences of labeled inputs $(\alpha, c ? v)$ and outputs $(\alpha, c ! v)$ to indicate the process involved in the communication. Given such a sequence $h$ and a channel $c \in C$ we denote by $\operatorname{sent}(h, c)$ the sequence of values in $V a l$ sent to the channel $c$ and by $\operatorname{rec}(h, c)$ the sequence of values in $V a l$ received from the channel $c$.

A history $h$ is a (finite) sequence of labeled inputs $(\alpha, c ? v)$ and outputs $(\alpha, c ! v)$ which satisfies the following.

Prefix invariance: For every prefix $h^{\prime}$ of $h$ and channel $c$ we have that the sequence $\operatorname{rec}\left(h^{\prime}, c\right)$ of values delivered by $c$ is a prefix of the sequence $\operatorname{sent}\left(h^{\prime}, c\right)$ of values received by channel $c$.

Input ownership: For every prefix $h_{0} \cdot(\alpha, c ? v)$ of $h$, either the process $\alpha$ owns the input of the channel $c$ in $h_{0}$, or $h_{0}=h_{1} \cdot(\alpha, d ? c) \cdot h_{2}$ for some channel $d$ distinct from $c$ and $\alpha$ owns the input of the channel $c$ in $h_{2}$. A process $\alpha$ is said to be the owner of the input of a channel $c$ in a sequence $h$ if, for any channel $e$, there is no occurrence in $h$ of an output $(\alpha, e ! c)$, and for every occurrence in $h$ of an input $(\beta, c ? w)$ we have $\alpha=\beta$.

Output ownership: For every prefix $h_{0} \cdot(\alpha, c ! v)$ of $h$, either the process $\alpha$ owns the output of the channel $c$ in $h_{0}$, or $h_{0}=h_{1} \cdot(\alpha, d ? c) \cdot h_{2}$ for some channel $d$ (not necessarily distinct from $c$ ) and $\alpha$ owns the output of the channel $c$ in $h_{2}$. A process $\alpha$ is said to be the owner of the output of a channel $c$ in a sequence 
$h$ if for any channel $e$ there is no occurrence in $h$ of an output $(\alpha, e ! c)$, and for every occurrence in $h$ of an output $(\beta, c ! w)$ we have $\alpha=\beta$.

Input/output ownership essentially states that a process can communicate along a channel only if either it is the first user of that channel or it has received that channel via a preceding communication. Moreover, exclusive control over a channel is released only when that channel is outputted.

We can obtain the local information of a process from a given history as follows. For a history $h$, an internal state $s$, we write $s \simeq h$ if $s(\nu)=(\alpha, V)$ implies, for every channel $c$, both $s(c)=i n(h, \alpha, c)$ and $s(\bar{c})=\operatorname{out}(h, \alpha, c)$, where in $(h, \alpha, c)$ and out $(h, \alpha, c)$ denote the sequences of values received from and sent to the channel $c$ by the process $\alpha$ as recorded by the history $h$. Occurrences of $\gamma$ in those sequences will denote release of control of the channel $c$ by the process $\alpha$. More specifically, we have in $((\alpha, d ! c) \cdot h, \alpha, c)=\gamma \cdot$ in $(h, \alpha, c)$ and similarly for $\operatorname{out}((\alpha, d ! c) \cdot h, \alpha, c)$. Thus $s \simeq h$ basically states that the information about the communication behavior in the internal state $s$ is compatible with the information given by the history $h$. The compatibility of $h$ with respect to a set of internal states $X$ is defined below.

Definition 3. Let $h$ be a history and $X$ be a finite set of internal states. We say that $h$ is compatible with $X$ if the following two conditions hold:

1. for every $s \in X, s \simeq h$;

2. there exists a finite tree (the tree of creation) with $X$ as nodes such that

- if $s$ is the root of the tree then $s(\nu)=(\perp, V)$, for some $V \subseteq C$;

- if $s \in X$ with $s(\nu)=(u, V)$ then for all $v \in V$ there exists a unique direct descendent node $s^{\prime} \in X$ with $s^{\prime}(\nu)=(v, W)$, for some $W \subseteq C$.

The existence of a tree of creation ensures the uniqueness of the name of the created channels. It is worthwhile to observe that it is not sufficient to require disjointness of the names used by any two distinct existing processes, as this does not exclude cycles in the creation ordering (for example, two processes creating each other).

Let $h$ be a history compatible with a finite set of (local) states $X$. For each channel $c$ which appears in $X$, we denote by own $(h, c)$ and $o w n(h, \bar{c})$ the sequences of processes who had the ownership of the reference for inputting from and outputting to the channel $c$, respectively.

For a given set of (local) states $X$ there may be several histories, each of them compatible with $X$. The next theorem specifies the relevant information recorded in a history.

Theorem 1. Let $X$ be a finite set of (local) states, and $h_{1}$ and $h_{2}$ be two histories compatible with $X$. For all process id's $\alpha$ and channels $c$ the following holds:

1. in $\left(h_{1}, \alpha, c\right)=$ in $\left(h_{2}, \alpha, c\right)$ and out $\left(h_{1}, \alpha, c\right)=\operatorname{out}\left(h_{2}, \alpha, c\right)$;

2. $\operatorname{sent}\left(h_{1}, c\right)=\operatorname{sent}\left(h_{2}, c\right)$ and $\operatorname{rec}\left(h_{1}, c\right)=\operatorname{rec}\left(h_{2}, c\right)$;

3. own $\left(h_{1}, c\right)=\operatorname{own}\left(h_{2}, c\right)$ and $o w n\left(h_{1}, \bar{c}\right)=o w n\left(h_{2}, \bar{c}\right)$. 
This theorem states that the compatibility relation abstracts from the order of communication between different channels in a global history. For example, even the ordering between inputs and outputs on different channels is irrelevant. This contrasts with the usual models of asynchronous communicating nondeterministic processes $[14,15]$. This abstraction is made possible because of the restriction to confluent programs.

In order to formulate the main theorem of this paper we still need some more definitions. We say that a set $X$ of extended internal states is consistent if there exists a history $h$ compatible with $X$. Given a consistent set $X$ of extended internal states $s$, we denote by $\operatorname{con} f(X)$, the corresponding (final) configuration $\langle\tilde{X}, \sigma\rangle$. That is, $\tilde{X}$ consists of those pairs (nil, $\tilde{s}$ ) for which there exists $s \in X$ such that $\tilde{s}$ is obtained from $s$ by removing the additional information about the communicated values and the created channels. The global state $\sigma$ derives from a history $h$ compatible with $X$ in the obvious way (i.e. by mapping every channel $c$ such that $s(\nu)=(c, V)$ for some $s \in X$ and $V \subseteq C$, to the sequence obtained by deleting the prefix $\operatorname{rec}(h, c)$ from $\operatorname{sent}(h, c))$. Note that the above Theorem 1 guarantees that $\sigma$ is indeed well-defined.

Definition 4. We assume given $T_{i}$ and $R_{i}$, for $i=1, \ldots, n$, with $T_{i}$ a set of (extended) internal states and $R_{i}$ a set of triples of the form $(s, c, t)$, where $s$ is an extended internal state, $c$ is a channel and $t$ is a type (of the value to be read from $c$ in the state $s$ ).

We denote by $\bigsqcup_{i} T_{i}$ the set of final configurations conf $(X)$ such that the set $X$ of (extended) internal states is consistent and every state $s$ in $X$ belongs to some $T_{i}$. Additionally, for some state $s \in T_{0}$ we have $s(\nu)=\langle\perp, V\rangle$, for some $V \subseteq C$.

Analogously, by $\bigsqcup_{i}\left\langle T_{i}, R_{i}\right\rangle$ we denote the set of final configurations conf $(X)$ such that $X$ is consistent, and there exists a state $s$ in $X$ that does not belong to any $T_{i}$, and, finally, every state $s$ in $X$ either belongs to some $T_{i}$ or there exists a triple $(s, c, t) \in R_{i}$ such that either $\sigma(c)=\epsilon$ or the first value of $\sigma(c)$ is not of type $t$.

Abstracting from the control information, the set of configurations $\bigsqcup_{i}\left\langle T_{i}, R_{i}\right\rangle$ in fact describes all possible deadlock configurations, whereas $\bigsqcup_{i} T_{i}$ describes all the final configurations of successfully terminating computations of the given program. Finally, we are in a position to formulate the main theorem of this paper.

Theorem 2. Let $\rho=\left\{P_{0} \Leftarrow S_{0}, \ldots, P_{n} \Leftarrow S_{n}\right\}$ and $\mathcal{O}\left(S_{i}\right)=\left\langle T_{i}, R_{i}\right\rangle, i=$ $0, \ldots, n$. We have that

$$
\mathcal{O}(\rho)= \begin{cases}\bigsqcup_{i} T_{i} & \text { if } \bigsqcup_{i}\left\langle T_{i}, R_{i}\right\rangle=\emptyset \\ \delta & \text { otherwise. }\end{cases}
$$

Thus the observable behavior of a confluent $M a C$ program can be obtained in a compositional manner from the local semantics of the statements of each process description of the program. The information of the ready sets of each local semantics is used to determine if the program deadlocks. 


\section{Conclusion and Future Work}

To the best of the authors knowledge, we have presented a first state-based semantics for a confluent language for mobile data-flow networks which is compositional with respect to the abstract notion of observable considered in this paper. This notion of observable is more abstract than the bisimulation-based semantics for most action-based calculi for mobility $[16,10,7]$, and the trace-based semantics for state-based languages [12].

The proposed semantics will be used for defining a compositional Hoare logic for confluent $M a C$ programs along the lines of [5]. The fact that the order between the communications between different processes and the communication on different channels within a process is semantically irrelevant will in general simplify the correctness proofs.

\section{References}

1. G. Agha, I. Mason, S. Smith, and C. Talcott. A foundation for actor computation Journal of Functional Programming, 1(1):1-69, 1993.

2. R. Amadio, I. Castellani, and D. Sangiorgi. On Bisimulations for the Asynchronous $\pi$-calculus. Theoretical Computer Science, 195:291-324, 1998.

3. F. Arbab, F.S. de Boer, and M.M. Bonsangue. A coordination language for mobile components. In Proc. of SAC 2000, pp. 166-173, ACM press, 2000.

4. F. Arbab, I. Herman, and P. Spilling. An overview of Manifold and its implementation. Concurrency: Practice and Experience, 5(1):23-70, 1993.

5. F.S. de Boer. Reasoning about asynchronous communication in dynamically evolving object structures. To appear in Theoretical Computer Science, 2000.

6. M. Broy. Equations for describing dynamic nets of communicating systems. In Proc. 5th COMPASS workshop, vol. 906 of LNCS, pp. 170-187, 1995.

7. L. Cardelli and A.D. Gordon. Mobile ambients. In Proc. of Foundation of Software Science and Computational Structures, vol. 1378 of LNCS, pp. 140-155, 1998.

8. E.W. Dijkstra. A Discipline of Programming. Prentice-Hall, 1976.

9. M. Falaschi, M. Gabbrielli, K. Marriot, and C. Palamidessi. Confluence in concurrent constraint programming. In Theoretical Computer Science, 183(2), 1997.

10. C. Fournet and G. Gonthier. The reflexive chemical abstract machine and the join calculus. In Proc. POPL'96, pp. 372-385, 1996.

11. R. Grosu and K. Stølen. A model for mobile point-to-point data-flow networks without channel sharing. In Proc. AMAST'96, LNCS, 1996.

12. G.J. Holzmann. The model checker SPIN IEEE Transactions on Software Engineering 23:5, 1997.

13. G. Kahn. The semantics of a simple language for parallel programming. In IFIP74 Congress, North Holland, Amsterdam, 1974.

14. He Jifeng, M.B. Josephs, and C.A.R. Hoare. A theory of synchrony and asynchrony. In Proc. IFIP Conf. on Programming Concepts and Methods, 1990.

15. B. Jonsson. A fully abstract trace model for dataflow and asynchronous networks. Distributed Computing, 7:197-212, 1994.

16. R. Milner, J. Parrow, and D. Walker. A calculus of mobile processes, parts I and II. Information and Computation 100(1):1-77, 1992.

17. E.-R. Olderog and C.A.R. Hoare. Specification-oriented semantics for communicating processes. Acta Informatica 23:9-66, 1986. 